\title{
Effective Contact Measures
}

\author{
Submission ID: 92
}

\begin{abstract}
Contact area is an important geometric measurement in many physical systems. It is also difficult to compute due to its extreme sensitivity to infinitesimal perturbations. In this paper, we propose a new concept called an effective contact measure, which acts as a smooth version of contact area. Effective contact measures incorporate a notion of scale into the definition of contact area, allowing one to consider the degree of contact at different sizes. We show how effective contact measures can yield useful statistics for a number of applications, including analysis of multiphase materials and docking/alignment problems.
\end{abstract}

Key words: contact area, measure, curvature, approximation

\section{Introduction}

Let us say that a pair of solids, $S, T \subseteq \mathbb{R}^{n}$ are in contact if their closures intersect but not their interiors: ${ }^{1}$

$$
\begin{array}{r}
(\kappa S \cap \kappa T) \neq \emptyset \\
(\iota S \cap \iota T)=\emptyset
\end{array}
$$

If $S$ and $T$ are in contact, then define their contact area to be the $(n-1)$-Hausdorff measure of their intersection:

$$
\mu^{n-1}(S \cap T)
$$

The goal of this paper is to study some approximations of contact area and their computations. In these approximations, we will consider both perturbation of the shapes and the situtation where the shapes are nearly in contact (ie they may be slightly separated or interpenetrating, violating the strict contact condition).

\subsection{Motivation}

There are a number of reasons that one might be interested in contact area. As a motivating example, consider the problem of analyzing a sample of some multiphase material. In general, some of the phases may be insulated from one another, while others may be directly touching. The amount that any two phases are in contact has a direct physical significance in heat transfer[21], electrical resistance[20], material strength[15], mechanical wear[2] and

\footnotetext{
1 We use the symbols $\kappa, \iota, \delta$ to denote closure, interior and boundary operators respectively. We call a bounded set a solid if it is compact and regular; $S=\kappa \iota S$.
}

microscopic friction[5]. Another application of contact area is the solution of docking problems. Suppose we are given two solids - for example, pieces of a jigsaw puzzle - and say that we want to figure out how to align them such that they fit together while touching as much as possible. Finding such alignments could be used as a subproblem for higher level assembly and planning operations, like box packing[11] or recovering assembly constraints[9], for example.

\subsection{Challenges}

The main issue with the direct definition of contact area is that it is unstable with respect to small perturbations of the shapes involved. This seems to have been first noticed by Bowden[4], and is similar to the behavior of the surface area or curvature[19][6] of a solid. From a practical standpoint, this instability causes problems when using numerical optimization to find docking configurations. It also means the calculation of the contact area becomes highly dependent on the scale at which the objects involved are measured and represented, where results at finer scales may diverge greatly from those which are sampled more coarsely.

\subsection{Contributions}

Our proposed solution to these problems is to introduce a new a concept which we call an "effective contact measure". Effective contact measures allow us to consider contact area at different scales and in situations where ob- 
jects are nearly touching or even slightly penetrating. This avoids the use of highly sensitive calculations like surface area. The bulk of this paper is devoted to studying various formulations of effective contact measure. We also show many of these quantities can be computed efficiently using the Fourier transform which makes them attractive for problems like alignment or nesting. In the final portion of the paper, we discuss some of these applications in more detail and experimentally compare the behavior of our various constructions on several examples in 2D and 3D.

\section{Related Work}

In physics and mechanical engineering there has been a great deal of work in relating various physical quantities to contact area, as discussed in Section 1.1. But outside the applied sciences, the concept of contact area does not appear to have received much attention in its own right. Perhaps the most closely related set of ideas to our approach is the concept of a curvature measure[12,19], as defined in the field of geometric measure theory and integral geometry. However, a key difference is that curvature measures consider only the change of a single body within a fixed localizing region, while in a contact area measure we consider simultaneous perturbations of both sets.

To the best of our knowledge, the direct application of contact area to alignment or docking problems does not appear to have been investigated in either a theoretical or computational context. In spirit, the most closely related research to this work is cross correlation based alignment for protein docking[16,14]. These techniques model the alignment problem as one of maximizing the overlap of psuedo electric charge potentials defined over the surface of some molecule, with complex weights added to the interiors to penalize interference and reward docking. Variations of this idea are currently used in state of the art systems of finding probably docking configurations in drug discovery $[8,3]$. This same sort of operation is at the heart of our approach to contact area, though we do not use the same approach to defining the "electric fields" and give the operation a very different interpretation. Finally, it bears mentioning that Kazhdan used spherical harmonics to align pairs of 3D models up to a rotation[17] by cross correlating their surface normals. This technique can be interpreted as a special case of the Laplacian contact area we propose in the later section.

\section{Effective Contact Area}

\subsection{Basic Requirements}

We choose to cast our theory of effective contact measures in the language of bimeasures. The motivation for bimeasures comes from a desire to extend measures from one to many sets. At minimimum, a bimeasure satisfies the following definition:
Definition $1 A$ bimeasure is a real valued binary function $K$ on solids such that for all compact regular sets $S, T \subseteq \mathbb{R}^{n}$, $K$ satisfies the following axioms:

(i) (Null-Emptyset) $K(\emptyset, S)=K(S, \emptyset)=0$.

(ii) (Additive) Given $S_{1}, S_{2}, T_{1}, T_{2} \subseteq \mathbb{R}^{n}$ where $S_{1} \cap S_{2}=$ $\emptyset$ and $T_{1} \cap T_{2}=\emptyset$,

$$
\begin{gathered}
K\left(S_{1} \cup S_{2}, T_{1} \cup T_{2}\right)=K\left(S_{1}, T_{1}\right)+K\left(S_{1}, T_{2}\right)+ \\
K\left(S_{2}, T_{1}\right)+K\left(S_{2}, T_{2}\right) .
\end{gathered}
$$

We want to define bimeasures which capture some notion of contact size between a pair of solids. But a bimeasure is a very general type of structure, and so for our goals it is reasonable to impose some additional constraints upon the bimeasures and the class of solids that we will be investigating. Inspired by the ideas of Edalat and Lieutier[10], we would like to ensure that our functions are computable by convergent sequences of solids, for example in the Hausdorff topology. Hence the following two definitions.

Definition 2 A sequence $S_{0}, S_{1}, \ldots \subseteq \mathbb{R}^{n}$ converges to $S \subseteq$ $\mathbb{R}^{n}$ (in the Hausdorff topology) [13] if for any $\epsilon>0$, there exists some $k \in \mathbb{N}^{+}$such that for all $i>k$,

$$
S \subseteq S_{i} \oplus \epsilon B^{n} \text { and } S_{i} \subseteq S \oplus \epsilon B^{n}
$$

Where $\oplus$ denotes the Minkowski sum and $B^{n}=\left\{x \in \mathbb{R}^{n}\right.$ : $|x| \leq 1\}$ is the unit ball.

Definition 3 A bimeasure is continuous (in the Hausdorff topology) if for any pair of convergent sequences, $\left\{S_{i}\right\},\left\{T_{j}\right\}$ approaching $S, T$ respectively, we have:

$$
\lim _{i, j \rightarrow \infty} K\left(S_{i}, T_{j}\right)=K(S, T)
$$

Continuity is a necessary requirement for a bimeasure to be robustly computable[10]. Without this property, small perturbations in the input shapes can have arbitrarily large effects on the result. The desire for continuity is also the reason why we must look beyond the most naive measures of contact area. In fact one can see that the Hausdorff area of the intersection fails to be continuous from the following simple example. Given two plates which are separated by some finite distance $t$, they determines a sequence of solids which converge to a pair of plates that are exactly in contact. For any $t>0$, the two plates are not in contact and so $\mu^{n-1}(S \cap T)$ is 0 ; however in the limit where they touch it $\mu^{n-1}(S \cap T)$ jumps to the area of the intersection, and so the measure is obviously not continuous.

\subsection{Effective Contact Area}

The fact that the Hausdorff measure of the intersection of two solids is discontinuous is the main technical necessity for pursuing this research. Our proposed solution to this problem is to first replace the measure of the intersection with an integral of indicator functions, and then to apply smoothing that the resulting function is continuous. This smoothing introduces a concept of scale, where coarser scales correspond to more smoothing, while fine values give a sharper measure which in the limit converges to the exact contact area. A side effect of this smoothing is that we 
recover finite effective contact area in the case where the solids involved are not in strictly contact, that is they might not intersect or their interiors may be slightly overlapping. Formalizing this concept is the basis for our new definition of an effective contact measure,

Definition $4 A n$ effective contact measure is a family of bimeasures $\left\{K_{h}: h>0\right\}$ such that for $h>0$ each $K_{h}$ is continuous, and that for any pair of solids of finite reach which are in contact $^{2}$, there exists a real number $C>0$ such that,

$$
\lim _{h \rightarrow 0} K_{h}(S, T)=C \mu^{n-1}(S \cap T) .
$$

The parameter $h$ acts as a scaling variable, which controls the precision to which we are measuring contact area. The main advantage to this definition is that for all positive $h$, $K_{h}$ is continuous and so in principle we could find efficient algorithms for measuring/computing this quantity. But, it is important to note this definition does not fully constrain the specific type of contact measure, and so there are many valid choices for $K_{h}$. Each of these choices corresponds to a different notion of scale, and thus leads to distinct concepts of effective contact area. In the subsequent sections we will study a few possible constructions, and compare their results.

\subsection{Group Invariance}

In addition to continuity, another fairly basic requirement is postulating that our contact measure is preserved under some kind of symmetry:

Definition 5 Let $G$ be a group acting on $\mathbb{R}^{n}$. A bimeasure is $G$-invariant if for all $g \in G$

$$
K(S, T)=K(g S, g T)
$$

Typically, we will take $G=S E(n) \cong S O(n) \ltimes \mathbb{R}^{n}$ to be the group of rigid motions ${ }^{3}$. That is, we require that contact measures should not change under rigid transformations of coordinate systems. Given a $G$-invariant effective contact measure, and fixing the pair of solids $S, T$, we can treat the contact measure as purely a function of the relative configuration of between the two bodies. That is, we imagine leaving one body fixed and moving the other one by some motion. This motivates us to define the concept of kinematic (effective) contact area:

Definition 6 Given a $G$-invariant bimeasure $K$ and a pair of solids $S, T \subseteq \mathbb{R}^{n}$, we define the kinematic effective contact area of $K$ to be a function $\mathcal{C}_{S, T}^{K}: G \rightarrow \mathbb{R}$ such that,

$$
\mathcal{C}_{S, T}^{K}(g)=K(S, g T) \text {. }
$$

Note that this is not a symmetric definition as,

$$
\mathcal{C}_{S, T}^{K}(g)=\mathcal{C}_{T, S}^{K}\left(g^{-1}\right) .
$$

In practical terms, we would like to calculate this quantity at varying configurations as $S$ and $T$ move around.

\footnotetext{
2 See introduction

3 That is $S O(n)$ is the group of $n \times n$ orthogonal matrices - aka ndimensional rotations - and $S E(n)$ is the group of and translations.
}

Finding configurations which maximize the contact area intuitively correspond to "good" alignments or tight fits. As a result, in parallel to the analysis of the contininuity of our proposed effective measures, we will consider the additional problem of computing $\mathcal{C}_{S, T}^{K}$.

\section{Examples of Effective Contact Measures}

In summary, we want to find families of continuous $S E(n)$-invariant bimeasures which have simple kinematic effective contact areas and converge to the area of the intersection in the limit. As we discussed earlier, these effective contact measures can be interpreted as defining contact area at various scales. Before starting this section, it is advised that the reader skim Appendices A,B to review some of the basic concepts that we will use in the following sections. Appendix A explains that group-invariant volumetric intersection is a continuous bimeasure that may be computed efficiently as a convolution. Appendix ${ }^{6} \mathrm{~B}$ is a standard definition of Minkowski content $M$ that is commonly used to measure fractal boundaries[13].

\subsection{Parallel Intersection}

We call the first effective contact measure that we shall consider the parallel intersection. The main idea behind this definition is a modification of the Minkowski content $M$ as defined in see Appendix B. For solids $S$ and $T$ which are in contact, it is true that $S \cap T=\delta(S \cap T)$ and so:

$\mu^{n-1}(S \cap T)=M(S \cap T)=\lim _{h \rightarrow 0} \frac{\mu\left((S \cap T) \oplus h B^{n}\right)-\mu(S \cap T)}{h \mu^{n-1}\left(B^{n-1}\right)}$.

This expression is not continuous in $S$ and $T$, but writing the contact area in terms of the Minkowski content is revealing. The underlying problem is that the numerator on the right hand side contains both an intersection and a dilation operator, and that these operations do not distribute. Practically speaking, this means that computing the intersection area is very difficult since we can't write it as a convolution as we could in the case of intersection volume. However, it does motivate the following definition: Definition 7 Define the parallel intersection area of two sets, $S, T$ to be:

$$
K_{h}^{p a r}(S, T)=\frac{\mu\left(\left(S \oplus h B^{n}\right) \cap\left(T \oplus h B^{n}\right)\right)-\mu(S \cap T)}{h \mu^{n-1}\left(B^{n-1}\right)}
$$

This definition is not in general the same as contact area, but in the limit where $h \rightarrow 0$ it does converge to contact area in the following sense:

Theorem $8 K_{h}^{p a r}$ is an effective contact measure for polytopes.

PROOF. That $K_{h}^{p a r}$ is continuous follows from Thm. 11 in Appendix A since, 


$$
\begin{aligned}
& K_{h}^{\text {par }}(S, T)= \\
& \frac{1}{h \mu^{n-1}\left(B^{n-1}\right)}\left(K^{i n t}\left(S \oplus h B^{n}, T \oplus h B^{n}\right)-K^{i n t}(S, T)\right) .
\end{aligned}
$$

The hard part is to show that $K_{h}^{\text {par }}$ converges to the Minkowski content of the intersection as $h$ goes to 0 , or in other words that,

$$
\lim _{h \rightarrow 0} \mu\left(\left(S \oplus h B^{n}\right) \cap\left(T \oplus h B^{n}\right)\right)=\lim _{h \rightarrow 0} \mu\left((S \cap T) \oplus h B^{n}\right) .
$$

Because $(S \cap T) \subseteq\left(S \oplus h B^{n}\right) \cap\left(T \oplus h B^{n}\right)$,

$\lim _{h \rightarrow 0} \mu\left((S \cap T) \oplus h B^{n}\right) \leq \lim _{h \rightarrow 0} \mu\left(\left(S \oplus h B^{n}\right) \cap\left(T \oplus h B^{n}\right)\right)$,

so it remains to show then that for small enough $h$,

$$
\mu\left(\left(S \oplus h B^{n}\right) \cap\left(T \oplus h B^{n}\right)\right) \leq \mu\left((S \cap T) \oplus h B^{n}\right)+O\left(h^{2}\right) .
$$

To prove Eqn. 4, decompose the boundary of $S$ and $T$ into finitely many convex cells. By the hypothesis that $S$ and $T$ are in contact, $S \cap T=\delta S \cap \delta T$, so for small enough $h$,

$\mu\left(\left(S \oplus h B^{n}\right) \cap\left(T \oplus h B^{n}\right)\right)=\mu\left(\left(\delta S \oplus h B^{n}\right) \cap\left(\delta T \oplus h B^{n}\right)\right)$.

Let $\operatorname{skel}_{n-2} \delta S$ be the $n-2$ skeleton $^{4}$ of $\delta S$ and $\operatorname{skel}_{n-2} \delta T$ be the $n-2$ skeleton of $T$, and let the relative interior of each boundary face of $S$ and $T$ be $F_{i}, G_{j}$ respectively. Then,

$$
\delta S=\operatorname{skel}_{n-2} \delta S \cup \bigcup_{i} F_{i} .
$$

Where each $F_{i}, G_{j}$ is a pairwise disjoint open cell. By the distributivity of Minkowski addition,

$$
\delta S \oplus h B^{n}=\left(\operatorname{skel}_{n-2} \delta S \oplus h B^{n}\right) \cup \bigcup_{i} F_{i} \oplus h B^{n} .
$$

Using this, we split into two terms,

$$
\mu\left(\left(\delta S \oplus h B^{n}\right) \cap\left(\delta T \oplus h B^{n}\right)\right) \leq P_{1}+P_{2},
$$

where

$$
\begin{aligned}
& P_{1}=\mu\left(\operatorname{skel}_{n-2} \delta S \oplus h B^{n}\right)+\mu\left(\operatorname{skel}_{n-2} \delta T \oplus h B^{n}\right) \\
& P_{2}=\sum_{i, j} \mu\left(\left(F_{i} \oplus h B^{n}\right) \cap\left(G_{j} \oplus h B^{n}\right)\right) .
\end{aligned}
$$

By the Minkowski-Steiner forumla, the measure of the $n-2$ skeleton of a polytope is proportional to $O\left(h^{2}\right)$ times the mean curvature of the set,

$$
\mu\left(\operatorname{skel}_{n-2} \delta S \oplus h B^{n}\right) \in O\left(h^{2}\right) .
$$

Therefore,

$$
P_{1} \leq O\left(h^{2}\right) \text {. }
$$

For $P_{2}$, consider a pair of cells $F_{i}$ and $G_{j}$ and let $\operatorname{rel} \delta F_{i}$ denote the relative boundary of $F_{i}$. Then,

$$
\begin{aligned}
\mu\left(\left(F_{i} \oplus h B^{n}\right) \cap\left(G_{j} \oplus h B^{n}\right)\right) & \leq \mu\left(\left(F_{i} \cap G_{j}\right) \oplus h B^{n}\right) \\
& +\mu\left(\operatorname{rel} \delta F_{i} \oplus h B^{n}\right) \\
& +\mu\left(\operatorname{rel} \delta G_{j} \oplus h B^{n}\right) .
\end{aligned}
$$

\footnotetext{
4 The $k$ skeleton of a polytope is the union of all closed $k$ dimensional cells in the polytope.
}

And since $\operatorname{rel} \delta F_{i} \subseteq \operatorname{skel}_{n-2} S$,

$\mu\left(\left(F_{i} \oplus h B^{n}\right) \cap\left(G_{j} \oplus h B^{n}\right)\right) \leq \mu\left(\left(F_{i} \cap G_{j}\right) \oplus h B^{n}\right)+O\left(h^{2}\right)$.

Putting this all together,

$\mu\left(\left(S \oplus h B^{n}\right) \cap\left(T \oplus h B^{n}\right)\right) \leq \mu\left((S \cap T) \oplus h B^{n}\right)+O\left(h^{2}\right)$.

And so we conclude that,

$\lim _{h \rightarrow 0} \mu\left(\left(S \oplus h B^{n}\right) \cap\left(T \oplus h B^{n}\right)\right)=\lim _{h \rightarrow 0} \mu\left((S \cap T) \oplus h B^{n}\right)$.

We conjecture that this result holds in much more generality than for just polytopes, however in practice it should be sufficient for our purposes here. The main value in this formulation is Eqn. 3. Using the results in Appendix A, we can also express $\mathcal{C}_{S, T}^{K_{\epsilon}^{\text {par }}}$ for the group of translations (ie $\mathbb{R}^{n}$ ) as a correlation,

$$
\mathcal{C}_{S, T}^{K_{h}^{p a r}}=\frac{1}{h \mu^{n-1}\left(B^{n-1}\right)}\left(1_{S \oplus h B^{n}} * 1_{T \oplus h B^{n}}-1_{S} * 1_{T}\right) .
$$

In practical terms, this means that we can directly compute the parallel intersection at any finite scale $h$ by sampling in $O(n \log (n))$ time, where $n$ is the number of samples. While parallel intersection volume does represent a practical improvement over the exact contact area, it is by no means a perfect solution. In particular, it has the somewhat undesirable property that, as the penetration depth between the two solids increases, so to does the effective contact area. If our goal is to optimize the contact area, this increasing property will push the solids closer together, rather than rolling them along the boundary as we would desire. The reason for this behavior is that parallel intersection considers not only the intersection of the boundaries with themselves, but also the intersection of the boundary with the interior of each set.

\section{2. $\epsilon$-Boundary}

One direct way to improve on the previous result is to simply remove the interiors from the measure, which is the basis for our idea of the $\epsilon$-boundary measure and is defined as follows:

$$
K_{h}^{b n d}(S, T)=\frac{\mu\left(\left(\delta S \oplus h B^{n}\right) \cap\left(\delta T \oplus h B^{n}\right)\right)}{h \mu^{n-1}\left(B^{n-1}\right)}
$$

We now show that $K_{h}^{b n d}$ satisfies the basic requirements of a contact measure.

Theorem $9 K_{h}^{b n d}$ is an effective contact measure for polytopes.

PROOF. To see that this is true, we can rewrite

$$
\delta S \oplus h B^{n}=\left(S \oplus h B^{n}\right) \backslash\left(S \ominus h B^{n}\right)
$$

Here we are using $\ominus$ to denote Minkowski subtraction (aka erosion), that is

$$
S \ominus h B^{n}=\left(S^{C} \oplus h B^{n}\right)^{C}
$$


Because $S \ominus h B^{n} \subset S \subset S \oplus h B^{n}$, we can simplify the expression for $K_{h}^{b n d}$,

$\mu\left(\left(\delta S \oplus h B^{n}\right) \cap\left(\delta T \oplus h B^{n}\right)\right)=$

$\mu\left(\left(S \oplus h B^{n}\right) \cap\left(T \oplus h B^{n}\right)\right)-\mu\left(\left(S \oplus h B^{n}\right) \cap\left(T \ominus h B^{n}\right)\right)$

$-\mu\left(\left(S \ominus h B^{n}\right) \cap\left(T \oplus h B^{n}\right)\right)+\mu\left(\left(S \ominus h B^{n}\right) \cap\left(T \ominus h B^{n}\right)\right)$

And so the continuity and invariance properties of the measure follow from the properties of the erosion operator. Similarly, for $S$ and $T$ in contact and with finite reach, and for sufficiently small $h$, we have

$$
\begin{aligned}
& \mu\left(\left(S \oplus h B^{n}\right) \cap\left(T \ominus h B^{n}\right)\right)=0 \\
& \mu\left(\left(S \ominus h B^{n}\right) \cap\left(T \oplus h B^{n}\right)\right)=0 \\
& \mu\left(\left(S \ominus h B^{n}\right) \cap\left(T \ominus h B^{n}\right)\right)=0
\end{aligned}
$$

As a result, for solids which are in contact the infinitesimal $\epsilon$-boundary contact measure reduces to the parallel contact, and so by Thm. 8 we have that

$$
\lim _{h \rightarrow 0} K_{h}^{b n d}(S, T)=\mu^{n-1}(S \cap T) .
$$

As a result, $K_{h}^{b n d}$ is an effective contact measure.

Eqn. 9 also contains the basic tools that we need to apply $K^{b n d}$ to alignment problems. It follows that over the group of translations,

$$
\mathcal{C}_{S, T}^{K_{h}^{b n d}}=c\left(1_{S \oplus h B^{n}}-1_{S \ominus h B^{n}}\right) *\left(1_{T \oplus h B^{n}}-1_{T \ominus h B^{n}}\right),
$$

where, $c=\frac{1}{h \mu^{n-1}\left(B^{n-1}\right)}$ is a normalization constant. In the case of shapes which are in contact, the $\epsilon$-boundary behaves similarly to the parallel contact area, however it is better behaved for interpenetrating solids.

\subsection{Laplacian}

The $\epsilon$-boundary is nice in that it does not become arbitrarily large as the solids interpenetrate; however it does not take into account the relative orientations of the surfaces. For example, alignment of interior faces counts equally as the alignment of the boundary when the solids are interpenetrating. A more minor issue is that calculating the dilation with a ball requires an extra $O(n)$ operation and so it would be better if we could avoid it. In this section we solve both of these problems by introducing the Laplacian contact area which we define as

$$
K_{h}^{\triangle}(S, T)=-\int_{\mathbb{R}^{n}} \triangle e^{-\frac{|x|^{2}}{h^{2}}}\left(1_{S} * 1_{T}\right)(x) d x,
$$

where $\triangle=\sum_{i=0}^{n-1} \frac{\partial^{2}}{\partial x_{i}^{2}}$ denotes the Laplace operator. The motivation for this definition is that we can imagine integrating the point-wise dot product of the surface normals of the two sets over their boundary. This is conceptually similar to the technique in [17] for aligning meshes via spherical harmonics except we operate in the ambient space instead of on the Gauss map. Eqn. 11 arises from smoothing the surface normals by a Gaussian, so that the surface normal can be properly defined ${ }^{5}$

Theorem $10 K_{h}^{\triangle}$ is an effective contact measure.

PROOF. Suppose $S$ and $T$ are solids with finite reach that are in contact. Then the contact area between $S$ and $T$ may be written as:

$$
\mu^{n-1}(\delta S \cap \delta T)=\int_{\delta S \cap \delta T}-n_{S}(x) \cdot n_{T}(x) d x,
$$

where $n_{S}$ and $n_{T}$ are the surface normals to $n_{S}$ and $n_{T}$ at the point $x$ for $S$ and $T$ respectively. For a solid with finite reach, the surface normal is the weak derivative of the indicator function, and so we can rewrite $n_{S}$ and $n_{T}$ as a gradient using a limit of nascent delta functions (which we select to be Gaussian),

$$
n_{S}(x)=\lim _{h \rightarrow 0} \int_{\mathbb{R}^{n}} \nabla_{t} e^{-\frac{|t|^{2}}{h^{2}}} 1_{S}(t-x) d t .
$$

So,

$$
\lim _{h \rightarrow 0} \iiint_{\mathbb{R}^{n}}-\left(\nabla_{t} e^{-\frac{|t|^{2}}{2 h^{2}}} 1_{S}(y-t)\right) \cdot\left(\nabla_{u} e^{-\frac{|u|^{2}}{2 h^{2}}} 1_{S}(y-u)\right) d y d t d u .
$$

By the commutativity of convolution and differentiation, and the closure of Gaussian distributions under convolution, this reduces to the desired limit,

$$
\mu(\delta S \cap \delta T)=\lim _{h \rightarrow 0}-c \int_{\mathbb{R}^{n}} \triangle e^{-\frac{|t|^{2}}{h^{2}}}\left(1_{S} * 1_{T}\right)(t) d t,
$$

where $c$ is a constant scale factor.

$K^{\triangle}$ behaves similarly to the $\epsilon$-boundary contact measure, with the additional property that when the surfaces are misaligned it returns a negative value. But the greatest advantage of the Laplacian contact is that it's kinematic representation is much simpler,

$$
\mathcal{C}_{S, T}^{K_{h}^{\Delta}}(R, x)=-\left(\triangle e^{-\frac{t^{2}}{h^{2}}} * 1_{S} * 1_{R T}\right)(x) .
$$

We can simplify this even further by taking a Fourier transform, reducing the Laplacian contact area to a simple Fourier multiplier of the correlation of the the shapes.

\section{Comparisons}

In summary, we have given three effective contact measures: (i)Parallel intersection, (ii) $\epsilon$-boundary, and (iii) Laplacian content. It may be instructive to compare these measures in terms of their formal properties to Hausdorff measure of the intersection,

$$
K^{\text {haus }}(S, T)=\mu^{n-1}(S \cap T),
$$

and the Minkowski content,

$$
K^{\text {mink }}(S, T)=M(S \cap T),
$$

\footnotetext{
5 That is the resulting smoothed normal field is in $H^{1}$ so that the dot product is well defined in the ambient space.
} 
even though these are not effective contact measures. The formal properties of all these bimeasures are summarized in the following table.

\begin{tabular}{|c||c|c|c|}
\hline Measure & Bounded & Positive & Continuous \\
\hline \hline Hausdorff Measure & - & $\checkmark$ & - \\
\hline Minkowski Content & - & $\checkmark$ & - \\
\hline Parallel Contact & $\checkmark$ & $\checkmark$ & $\checkmark$ \\
\hline$\epsilon$-Boundary Contact & $\checkmark$ & $\checkmark$ & $\checkmark$ \\
\hline Laplacian Contact & $\checkmark$ & - & $\checkmark$ \\
\hline
\end{tabular}

Here we rely on standard definitions. A bimeasure is positive if $B(S, T) \geq 0$ for all $S, T \subseteq \mathbb{R}^{n}$, and a bimeasure is bounded if $B(S, T)<\infty$ for all compact $S, T$. We defined continuity in Section 3.1.

In the rest of this section, we will compare the three effective contact measures empirically. by observing their behavior in typical application scenarios.

\subsection{Contact area under translation}

To communicate a more intuitive picture of the behavior of the three effective contact measures, consider the following situation. Suppose that we have two unit disks in $\mathbb{R}^{2}$ with centers $t$ units aparts. Then we can consider their effective contact area as a function of this distance $t$ :

$$
f_{K}(t ; h)=\mathcal{C}_{B^{2}, B^{2}}^{K_{h}}(t e),
$$

where $e$ is some aribitrary unit vector. Taking various choices for $K$ then lets us compare the contact measures visually at some scale in Fig. 1.

There are several interesting things to note about these plots. At the point $t=0$ the two balls completely overlap. In the parallel boundary and epsilon contact, the result is that they are completely aligned and so the contact area is proportional to the perimeter of a unit disk, or $2 \pi$ units, while in the Laplacian contact area we get a negatively oriented result. In general, when $t<1$ the two spheres are overlapping and at $t>1$ they are separated. At $t=1$ the two disks are exactly tangent to one another. While their exact contact area is 0 , for finite $h$ we get an effective contact area that is roughly proportional to $h$ in all cases, though the exact constant of proportionality varies somewhat.

Finally, it is interesting to observe the effect of $h$ on the computation. At large $h$ the contact area is much smoother as a function of distance, while at smaller $h$ the contact area becomes sharper but also accumulates more noise. This is most obvious in the Laplacian contact measure where integration artefacts appear even at $h=0.0625$. The $\epsilon$ boundary and parallel contact measures fare somewhat better, though they are more expensive to compute. One useful feature of the Laplacian contact is that the important features are generally more localized. As a result, it tends to give better information at larger scales. On the other hand,

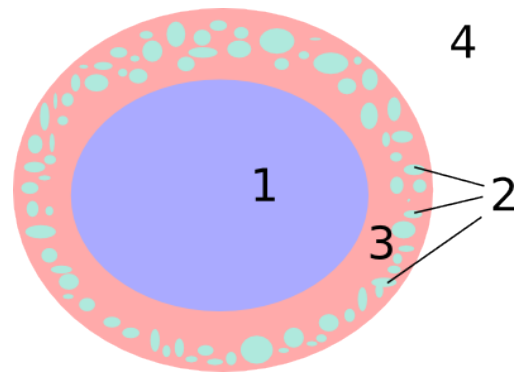

Fig. 3. An illustration of a multiphase material. In this image there are 4 separate phases including the void which have been labeled. We can use the various forms of effective contact measure to calculate the relative contact area between the different phases at various scales.

it is less robust than the other measurements for very fine contacts.

For computing exact contact area at small scales, it seems that the $\epsilon$-boundary is the best option. Not only does it have reasonably well localized features, but it also gives somewhat stable results in the case of partial interference. The only drawback is that it is slightly more expensive to calculate than the parallel boundary. In the situation where neither shape is interfering, the parallel and $\epsilon$-boundary measures give identical results, and so the choice of which measure to use is completely arbitrary.

The visual interpretation of the effective contact measures as a function of configuration parameters extends to higher dimensions. For example, in $2 \mathrm{D}$, the plot of field $\mathcal{C}_{S, T}^{K_{h}}$ as a function of relative position of the two shapes is similar to a cross correlation of the two shapes. This is shown in Fig. 2. The way to interpret the plots of $\mathcal{C}_{S, T}^{K_{h}}$ is that at each $\mathrm{x}, \mathrm{y}$ coordinate in the image the intensity is proportional to the effective contact area. One main difference is that the parallel boundary grows arbitrarily large when the solids penetrate, while the main difference between the Laplacian and $\epsilon$-Boundary measures is that the Laplacian contact becomes negative when the solids are oriented in an opposite direction.

\subsection{Contact area in multiphase materials}

To compare the role of scale in our proposed measures of contact area, we apply them to a simplified illustration of a multiphase material, Fig. 3. Though this example is synthetic, similar multiphase structures do appear in nature, in systems like trabecular bone or geological specimens.

In Fig. 3 the parallel contact and epsilon boundary give identical results since the interiors of the phases are all disjoint in these examples. For both of these measures, the peak of the contact area between any two phases corresponds roughly to the distance between them. For phases which are initially in contact with smooth boundaries, such as phase 1 and 3, the effective contact area is relatively flat for some time, and begins to taper off after the effective contact area surpasses the reach of the sets. It is also interesting to examine the effective contact area for phases with many small features, such as phase 2 . At small scales 

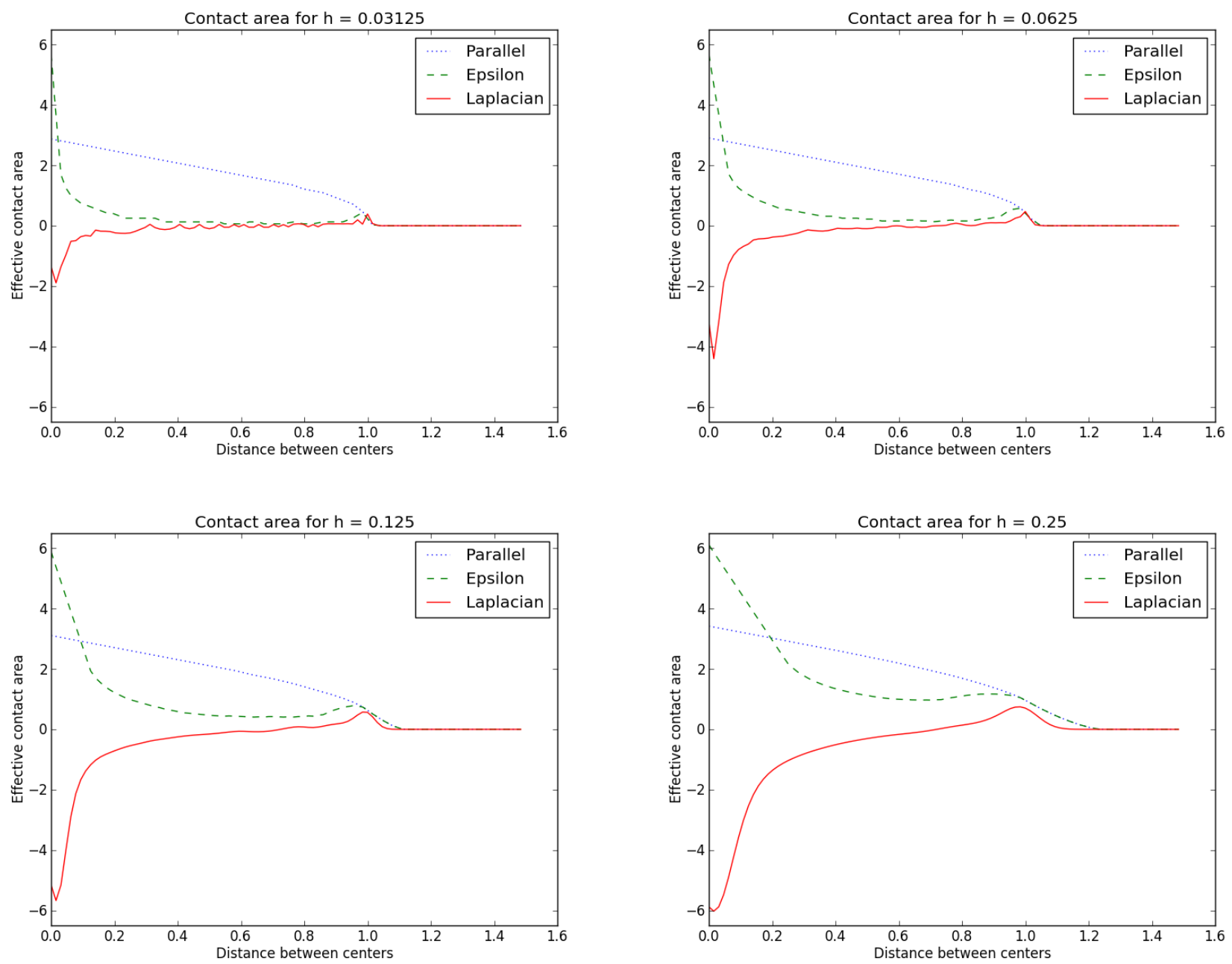

Fig. 1. Plots of $f_{K}(t ; h)$, which is the contact area for a pair of discs as a function of the distance between their centers, for varying scales, $h$ and for the parallel, $\epsilon$-boundary and Laplacian contact measures.

the effective contact area is very large, while it eventually decreases over time.

The Laplacian contact on the other hand has somewhat different behavior. While on the whole the location of the peaks in the Laplacian contact area are similar to the parallel and epsilon boundary, they tend to fall off sooner. One interesting feature is that the effective contact area between phase 2 and phase 3 becomes negative at large scales. This is because phase 2 is more or less homogenously distributed through phase 3 , and so at larger scales it is effectively contained within the outside material. Checking for a similarly shaped peak might be a useful technique to detect a particle phase which is distributed within a material matrix. Another interesting feature is the interaction between phase 3 and the phases 4 . At intermediate scales the the tiny holes cut out by phase 2 cause it to have a larger effective contact area, while at larger scales these average out and the area eventually stabilizes before ultimately decaying. This spike is not present in the effective contact area between 1 and 3 since there is a small gap which is on the order of the hole size and between phase 2 and phase 1 .

\section{Alignment}

Finally, we apply our effective contact measures to the problem of aligning two solids. Following section 3.3, we consider alignment as finding a configuration which locally maximizes some kinematic effective contact measure. The intuition for this is that if the surface of two solids are aligned, then any small perturbation of their configuration should move them farther apart and thus decrease the contact area. Of course the surfaces themselves may not precisely fit, especially if they are obtained from sampled data and so we must select some value for the scale parameter. The precise choice of this parameter depends on the feature size of the object which is being docked.

Finding an alignment requires a search over the relative configuration space of the pair of bodies. For each of the contact measures proposed, we showed that the kinematic contact area can be computed under translational motion using a Fourier transform. Doing this requires first voxelizing the shapes and then applying the FFT/convolution theorem. Finding the maximum configuration then amounts 

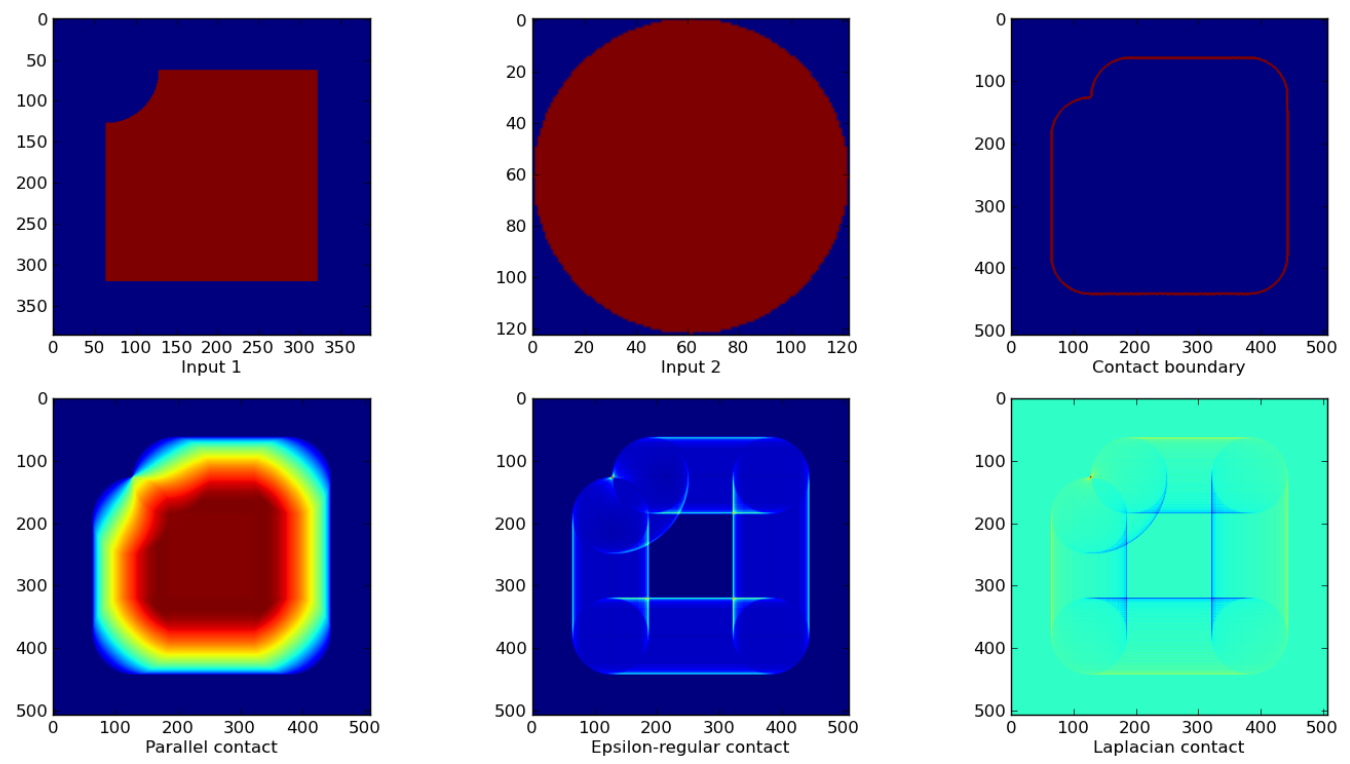

Fig. 2. 2D effective contact for the a pair of shapes. From left-to-right, in the top row the first two images show a pair of shapes, and the top-right image is the boundary of their configuration space obstacle. In the bottom row, we see the perimeter of their contact continued beyond the boundary into the interior region and beyond using the parallel contact, $\epsilon$-boundary, and Laplacian contact measures. Note that on the boundary all 3 measures give similar results, however in the interior region the results are very different. The color scale is calibrated such that red indicates a larger contact area, while blue is smaller. Note that each of these plots is using a separate scale. In the case of the epsilon and parallel contact, blue 0 while for the Laplacian contact area the 0 level is green and blue indicates a negative value.

to a scan over the output signal. In the worst case, the complexity of this operation is $O(n \log (n))$, where $n$ is the number number of samples.

To illustrate this method, several different problems were considered. Fig. 5 shows the Laplacian contact area between a pair of jigsaw puzzle pieces as a function of their relative translation. Higher values represent greater contact area. As can be seen from the plot, the spike on the right hand side is the greatest contact area value, which also happens to be the configuration corresponding to exactly docking the two pieces together.

However, there are also situations where maximizing contact area does not always give intuitive alignments. For example, in Fig. 6, the maximum contact area configuration is to place the two Lego bricks side by side instead of the snap fit corresponding to placing one on top of the other. The reason for this is that the bottom of the Lego brick is hollow, and so the relative contact area of the snap fit configuration is much lower.

Finally, to illustrate the importance of scale, we take a natural example. The dolphin cranium and mandible[1] in Fig. 7 are first aligned at finer scale, which produces incorrect results. Increasing the scale parameter though allows for the imprecise fit between the teeth of cranium and mandible to become more apparent, and leads to a more accurate fit.
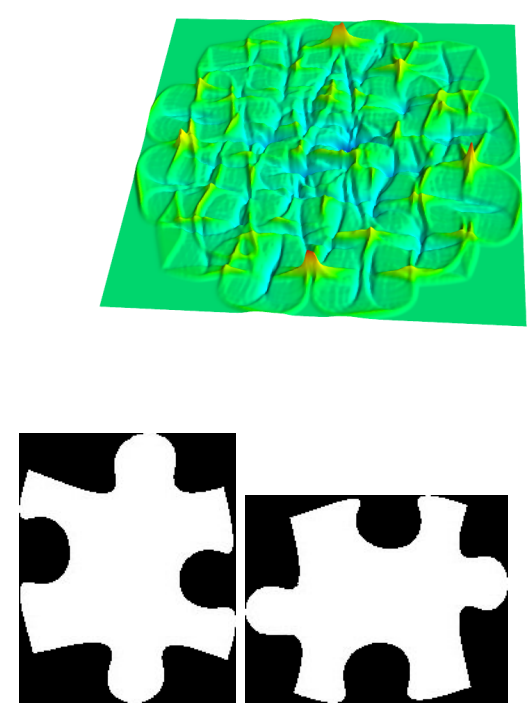

Fig. 5. Contact area for a pair of puzzle pieces. The plot (top) shows the Laplacian contact at varying relative translations, for the pair of puzzle pieces (bottom). Green is zero, red is positive and blue is negative. Note the peak on the right side represents the configuration where the two pieces are docked exactly.

\section{Conclusion}

In conclusion, we have introduced the concept of an effective contact measure and shown three different imple- 


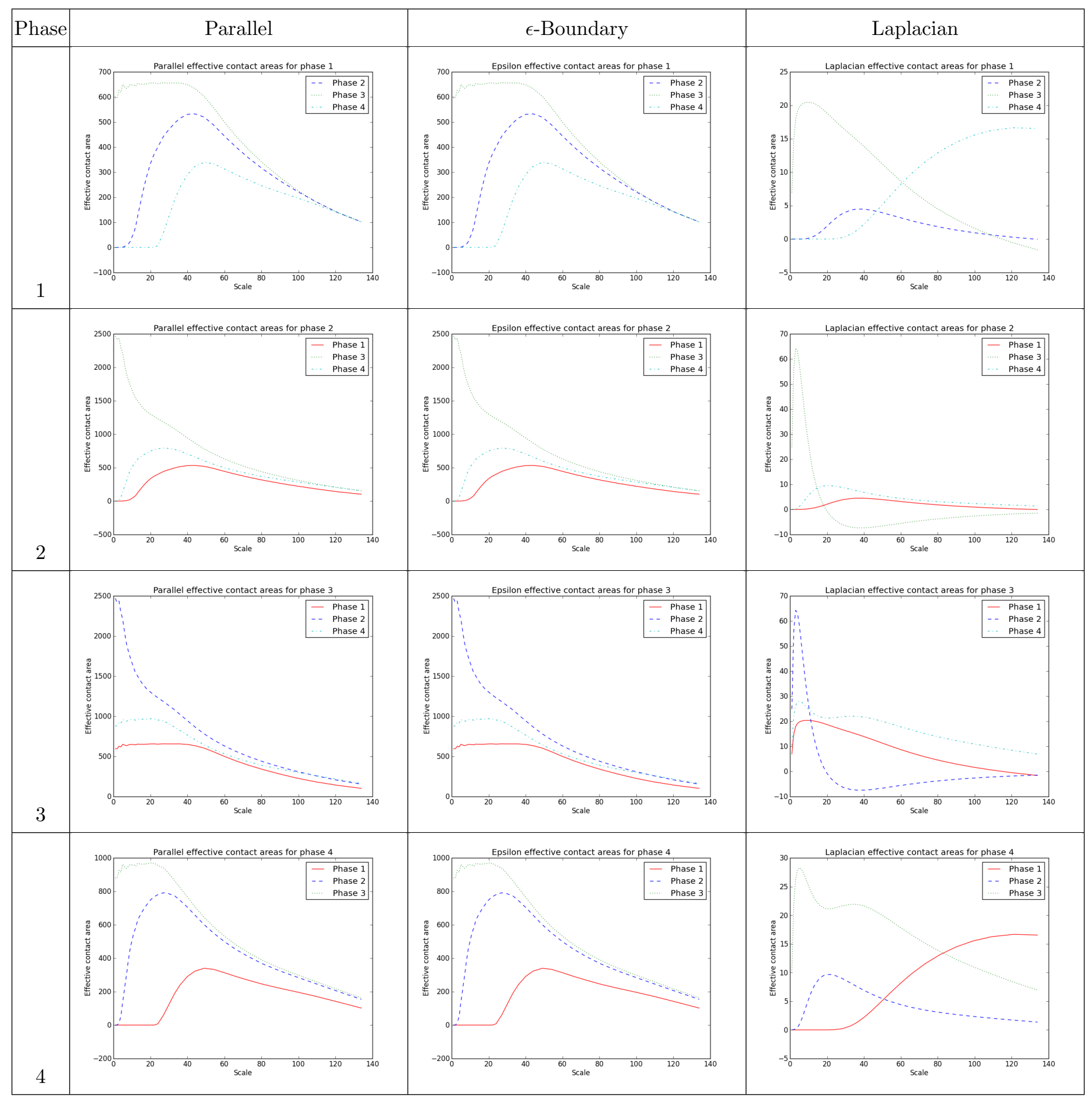

Fig. 4. Contact area between the different material phases of the illustration in Fig. 3. Each column shows the contact for a given measure, and each row is the contact for a given phase. The vertical axis in each plot is the effective measure and the horizontal axis is the scale parameter, $h$. Note that there is some symmetry here, since the contact area between phase 1 and 4 is the same as the contact are between phase 4 and 1 . Contact area between a phase and itself is not shown. 

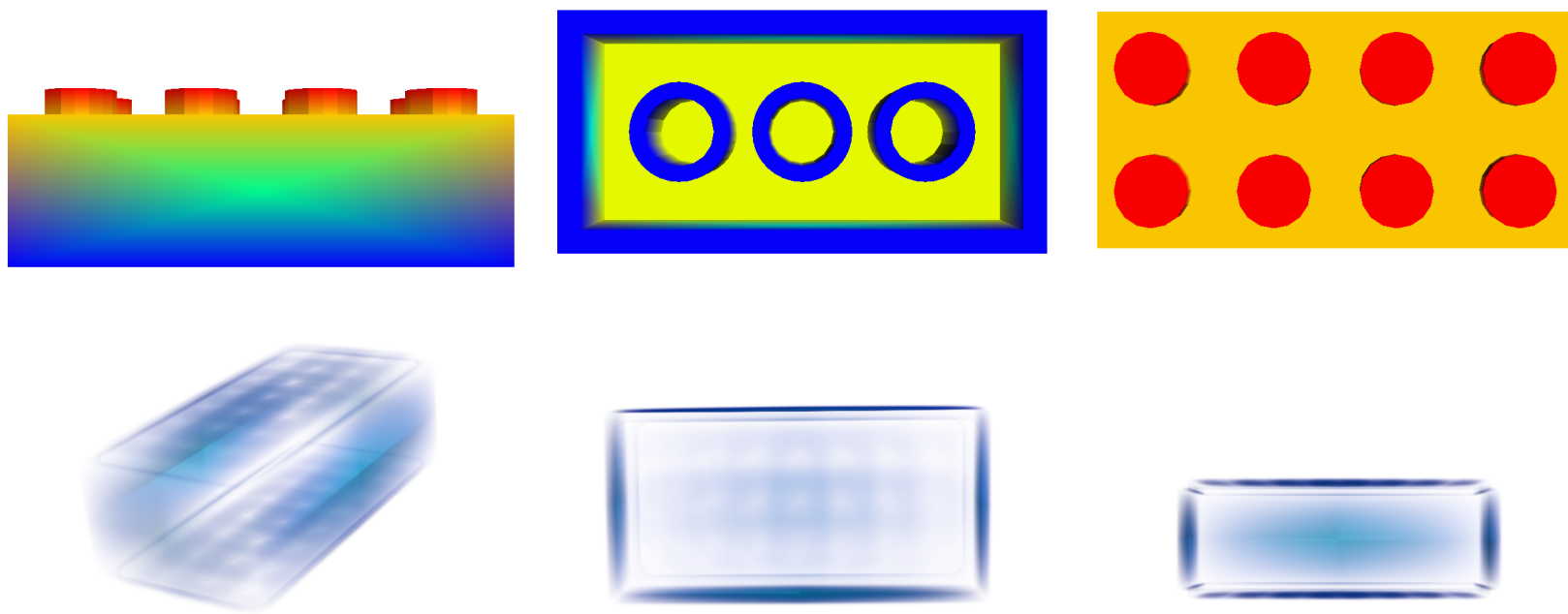

Fig. 6. Contact area between a pair of lego bricks. A lego brick (top row) is shown from different views. The bottom row shows the contact area of a lego brick with itself, where brighter colors indicate higher contact area. Note that the highest contact area is on the side of the brick. This is because the bottom of the lego brick is hollow, so the contact area for the snap fit of two blocks stacked on top of each other is lower. This represents a situation where contact area may not give what is intuitively the best fit.
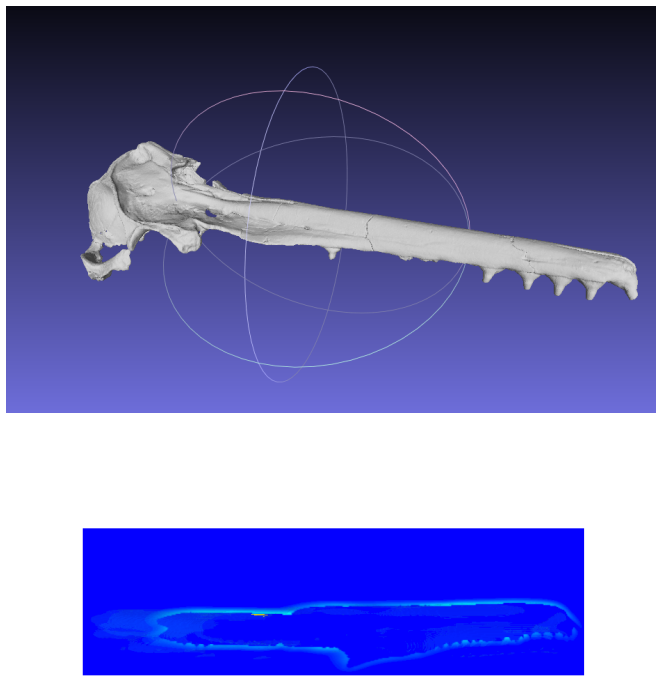

Cross section of Laplacian contact area with small $h$

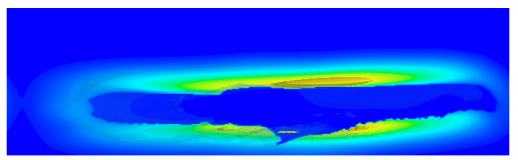

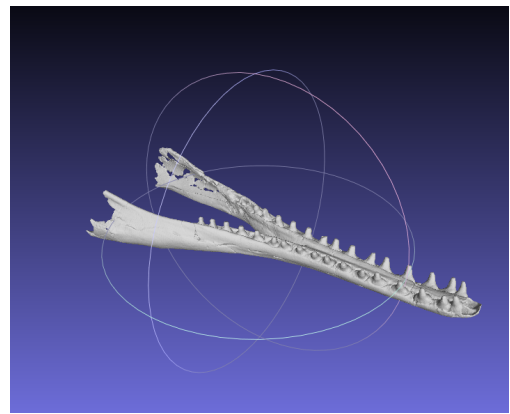

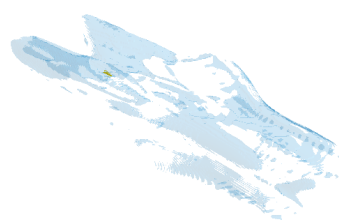

Isometric view of volume with small $h$

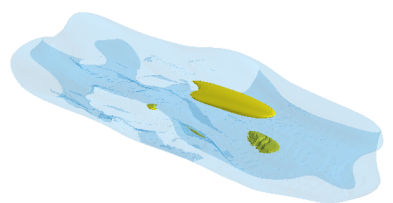

Cross section of Laplacian contact area for $5 h \quad$ Isometric view of Laplacian contact area for $5 h$

Fig. 7. Contact area for a fossilized dolphin cranium and mandible (data obtained from the Smithsonian Institute[1]). In the first row, contact area for a small $h$ are shown. In this situation, the contact area is uniform over the entire boundary of the configuration space obstacle. In the bottom row, a larger contact radius is used and variations in the contact area appear. One of these clusters appears in the configuration where the mandible is correctly assembled with the cranium, while the upper clusters correspond to configurations where the mandible is placed on top of the skull. 
mentations of this idea. Effective contact area represents a novel statistical measurement with an intuitive geometric meaning. In the future, it would be interesting to investigate the relationship between effective contact area and material properties of composites or heterogeneous structures. It seems very probable that the contact area between different microscopic phases could be important in determining the macroscopic physical properties of a complex microstructure. We also showed an application of effective contact area to docking problems and gave a simple FFT based algorithm for solving it. A logical extension of this idea would be to investigate using effective contact area in bin packing or nesting problems.

\section{Acknowledgements}

This research was supported in part by National Science Foundation grants CMMI-1029553 and CMMI-1344205. Any opinions, findings, and conclusions or recommendations expressed in this material are those of the authors and do not necessarily reflect the views of the National Science Foundation.

\section{References}

[1] River dolphin cranium and mandible, 2010.

[2] J. F. Archard. Contact and rubbing of flat surfaces. Journal of Applied Physics, 1953.

[3] C. Bajaj, R. Chowdhury, and V. Siddavanahalli. F2Dock: fast fourier protein docking. ACM Transactions on Computing Biology and Bioinformatics, 2011.

[4] F. P. Bowden and D. Tabor. The area of contact between stationary and moving surfaces. Proceedings of the Royal Society of London, 169(938):391413, 1939.

[5] Robert W. Carpick, D. Frank Ogletree, and Miquel Salmeron. A general equation for fitting contact area and friction vs load measurements. November 1999.

[6] Frederic Chazal, David Cohen-Steiner, Andre Lieutier, and Boris Thibert. Stability of curvature measures. In Computer Graphics Forum, volume 28, page 14851496, 2009.

[7] Gregory S. Chirikjian and Alexander B. Kyatkin. Engineering applications of noncommutative harmonic analysis: with emphasis on rotation and motion groups. CRC press, 2010.

[8] Gregory S. Chirikjian and Yunfeng Wang. Conformational statistics of stiff macromolecules as solutions to partial differential equations on the rotation and motion groups. Physical Review E, 62(1):880, 2000.

[9] GangWei Cui, YingXue Yao, and Yu Wang. Assembly process simulation of toleranced products. International Journal of Internet Manufacturing and Services, 2(3):248264, 2010.

[10] Abbas Edalat and Andr Lieutier. Foundation of a computable solid modeling. In Proceedings of the fifth ACM symposium on Solid modeling and applications, page 278284. ACM, 1999.

[11] Friedrich Eisenbrand, Stefan Funke, Andreas Karrenbauer, Joachim Reichel, and Elmar Schomer. Packing a trunk - now with a twist! In ACM Symposium on Solid Modeling and Applications, SM, page 197 206, 2005.

[12] H. Federer. Curvature measures. Transactions of the American Mathematical Society, 1959.

[13] Herbert Federer. Geometric Measure Theory.-Reprint of the 1969 Edition. Springer, 1996.
[14] H. A. Gabb, R. M. Jackson, and M. J. Sternberg. Modelling protein docking using shape complementarity, electrostatics and biochemical information. Journal of Molecular Biology, 1997.

[15] Joseph Gurland. The Measurement of Grain Contiguity in Twophase Alloys. PhD thesis, 1957.

[16] Ephraim Katchalski-Katzir, Isaac Shariv, Miriam Eisenstein, Asher A. Friesem, Claude Aflalo, and Ilya A. Vakser. Molecular surface recognition: determination of geometric fit between proteins and their ligands by correlation techniques. Proceedings of the National Academy of Sciences, 89(6):21952199, 1992.

[17] Michael Kazhdan. Approximate and efficient methods for optimal rotation alignment of 3D models. IEEE Transactions on Pattern Analysis and Machine Intelligence, 2006.

[18] M. Lysenko, S. Nelaturi, and V. Shapiro. Group morphology with convolution algebras. SPM 2010, 2010.

[19] J.-M. Morvan. Generalized Curvatures. Springer, May 2008.

[20] W. Shockley. Research and investigation of inverse epitaxial UHF power transistors. Technical Report A1-TOR-64-207, September 1964.

[21] James R. Welty, Charles E. Wicks, Robert E. Wilson, and Gregory L. Rorrer. Fundamentals of Momentum, Heat and Mass transfer. John Wiley and Sons, 5 edition, 2007.

\section{Appendix A. Intersection Volume, Correlation and Fourier Analysis}

Consider the problem of measuring the volume of intersection between two solids. That is, define the bimeasure:

$$
K^{i n t}(S, T)=\mu^{n}(S \cap T)
$$

It is easy to see that:

\section{Theorem $11 \quad 1 . K$ is a bimeasure.}

2. $K$ is invariant under rigid motions.

3. $K$ is continuous.

But more importantly, $\mathcal{C}^{K}$ can be computed efficiently using techniques from representation theory/Fourier analysis. More details can be found in [18],[7], though we shall summarize the story here briefly for the sake of making this document self contained, and because we will need this notation in the sequel. First, given a solid $S$ we define,

$$
1_{S}(x)=\left\{\begin{array}{l}
1 \text { if } x \in S \\
0 \text { otherwise }
\end{array} .\right.
$$

Using indicator functions, many set theoretic operations translate into operations on functions. For example,

$$
\begin{aligned}
1_{S \cap T} & =1_{S} 1_{T}, \\
\mu^{n}(S) & =\int_{\mathbb{R}^{n}} 1_{S}(x) d x .
\end{aligned}
$$

With this notation we can rewrite $K$ as an integral,

$$
K^{i n t}(S, T)=\int_{\mathbb{R}^{n}} 1_{S}(x) 1_{T}(x) d x .
$$

And so, 


$$
\mathcal{C}_{S, T}^{K^{i n t}}(g)=\int_{\mathbb{R}^{n}} 1_{S}(x) 1_{T}\left(g^{-1} x\right) d x .
$$

This integral is an example of a group convolution,

$$
\left(f *_{G} h\right)(y)=\int_{G} f(x) h\left(x^{-1} y\right) d x .
$$

In the case where $G=\mathbb{R}^{n}$ which is the group of translations, then this is just the cross correlation of $1_{S}$ and $1_{T}$, and so it can be computed directly using a fast Fourier transform. More explicitly,

$$
\mathcal{C}_{S, T}^{K^{i n t}}(y)=\int_{\mathbb{R}^{n}} 1_{S}(x) 1_{T}(x-y) d x=\left(1_{S} *_{\mathbb{R}^{n}} 1_{T}\right)(y) .
$$

That is equivalent to the following by the convolution theorem,

$$
\mathcal{C}_{S, T}^{K^{i n t}}(y)=\int_{\mathbb{R}^{n}} \widehat{1_{S}}(\omega) \overline{\widehat{1_{T}}}(\omega) e^{i\langle\omega, y\rangle} d \omega .
$$

Here we use $\widehat{\cdot}$ to denote the Fourier transform,

$$
\widehat{1_{S}}(\omega)=\int_{\mathbb{R}^{n}} 1_{S}(x) e^{-i\langle\omega, x\rangle} d x .
$$

\section{Appendix B. Minkowski Content}

Given a solid $S$ with finite reach, there are at least two ways to compute its surface area:

(i) It is the $(d-1)$ Hausdorff measure of the boundary:

$$
\mu^{n-1}(\delta S)
$$

(ii) It is the Minkowski content of the set:

$$
M(S)=\lim _{\epsilon \rightarrow 0} \frac{\mu\left(S \oplus \epsilon B^{n}\right)-\mu(S)}{\epsilon \mu^{n-1}\left(B^{n-1}\right)}
$$

Where $B^{n}=\left\{x \in \mathbb{R}^{n}:|x| \leq 1\right\}$ is the unit ball, and $\oplus$ denotes the Minkowski sum of the two sets. Intuitively, the Minkowski content measures the volume of the infintesimal parallel offset surface formed by sweeping a ball along $S$. For more information see the text by Morvan[19]. 